\title{
PULSATIONAL INSTABILITY AND THE ORIGIN \\ OF PLANETARY NEBULAE
}

\author{
William K. Rose* \\ (Physics Dept, Massachusetts Institute of Technology, Cambridge, Mass., U.S.A.)
}

The calculations that will be discussed in this paper were undertaken to determine if plausible theoretical arguments could be found to indicate that planetary nebulae represent the stage in the evolution of a star immediately before it becomes a white dwarf. The model for the formation of a planetary nebula that will be described may also be applicable for more massive stars than have been studied. However, since it is known that some population-II stars become planetary nebulae and since there is evidence that evolved population-II stars are less massive than $1 M_{\odot}$ (Christy, 1966; Hayashi et al., 1962; Faulkner, 1966; Faulkner and Iben, 1966; Schwarzschild and Härm, 1966) only stars whose masses are less than $1 M_{\odot}$ have been studied.

Recent investigations of models for horizontal branch stars (Hayashi et al., 1962; Faulkner, 1966; Faulkner and Iben, 1966; Schwarzschild and Härm, 1966) indicate that the horizontal branch is an evolutionary stage such that the nuclear-energy generation results from helium burning at the centre of the star and hydrogen burning in a shell. In its subsequent evolution the population-II star will exhaust its supply of helium in the core. Helium shell-burning will result and the star will evolve up the red-giant branch for the second time. The model for the formation of planetary nebulae that is considered in this paper assumes that instabilities that arise during this helium shell-burning stage of evolution are responsible for the formation of planetary nebulae.

A number of investigators have computed stellar models that demonstrate the presence of thermal instability during the helium shell-burning stage of stellar evolution (Schwarzschild and Härm, 1965; Weigert, 1965; Rose, 1966). This instability has been found for models of stars that range in mass from $0.5 M_{\odot}$ to $5 M_{\odot}$. The physical basis for this instability lies in the high temperature sensitivity of the $3 \alpha$ process and the fact that the helium burning takes place in a thin shell. The calculations show that after a star has become thermally unstable it undergoes a series of relaxation oscillations. During each oscillation the rate of nuclear-energy generation is very high for a short period of time ( $\approx 100$ years for a typical oscillation). In general these periods of high nuclear-energy generation will not lead to the significant acceleration of mass that would be required to explain mass loss. However, under suitable conditions,

* Supported in part by the National Aeronautics and Space Administration (NsG-496).

Osterbrock and O' Dell (eds.), Planetary' Nebulae, 390-395. "I.A.U. 
high rates of nuclear-energy generation can lead to pulsational instability (Rose, 1967a). Although the calculations have so far only shown this to be true for blue stars, it is the assumption of this model for the formation of a planetary nebula that thermal instability can lead to pulsational instability in a red giant and that the presence of pulsational instability can lead to the formation of a planetary nebula.

There is an important distinction between pulsational instability in a very blue star and what we will call pulsational instability in a red giant. In the case of the blue star the injection of nuclear energy takes place in a time that is long as compared with the time for sound to traverse the star. Therefore, the adiabatic theory of pulsations is appropriate. On the other hand, in the case of the red giant the injection of nuclear energy can take place in a time that is comparable with or shorter than the time for sound to traverse the star. Therefore, the adiabatic theory of pulsations is not appropriate.

Although the low velocities of expansion observed for planetary nebulae $(\approx 20 \mathrm{~km} /$ sec) as well as independent arguments that are discussed in this paper indicate that planetary nebulae originate from red giants (a point that was first made by Shklovsky, 1956, and later amplified by Abell and Goldreich, 1966), the observed nuclei of planetary nebulae are very blue and must be almost completely hydrogen-deficient. This apparent paradox can be understood if it is recalled that for a helium shell-burning red giant almost all the gravitational and thermal energy of the configuration resides within a dense core. The core is composed of an interior region of mostly degenerate carbon and oxygen and an outer less massive region of semi-degenerate and nondegenerate matter that is mostly helium. If by some mechanism the outer hydrogenrich envelope were removed from the helium shell-burning red giant, a blue star with a degenerate core that is surrounded by a less dense helium-rich region would remain. For this reason, it is of some interest to study helium shell-burning stars with pure helium envelopes as approximate representations for the nuclei of planetary nebulae.

Evolutionary sequences of models for helium shell-burning stars with pure helium envelopes have been computed up to the end of helium burning and into the whitedwarf state (Rose, 1966, 1967a ; L'Ecuyer, 1966). Thermal stability analysis (Schwarzschild and Härm, 1965; Rose, 1966) has shown that some of these computed models are unstable. These unstable models undergo relaxation oscillations. During each of the relaxation oscillations the rate of nuclear-energy generation attains a high value for a short interval. Models with masses of $0.53 M_{\odot}, 0.75 M_{\odot}$ and $0.95 M_{\odot}$ have been studied. The results of these calculations show that the maximum rate of nuclearenergy generation is attained during the final relaxation oscillation, i.e. at the end of helium burning, and therefore just before the star contracts toward the white-dwarf state. The general behavior of the oscillations indicates a gradual increase in amplitude followed by a sharp increase for the final one or two oscillations. The maximum rate of nuclear-energy generation $\left(\approx 3 \times 10^{7} L_{\odot}\right)$ was attained for the $0.75 M_{\odot}$ star. This appears to be the case primarily because the helium-burning shell attained a higher 
density $\left(\approx 3 \times 10^{4} \mathrm{gm} / \mathrm{cm}^{3}\right)$ before the temperature of the helium shell went below that necessary to maintain helium burning for the $0.75 M_{\odot}$ sequence as compared with the $0.53 M_{\odot}$ and $0.95 M_{\odot}$ sequences.

A sequence of models for a helium shell-burning $\operatorname{star}\left(M=0.6 M_{\odot}\right)$ with an initial hydrogen envelope of $0.006 M_{\odot}$ has been computed through a series of relaxation oscillations up to the end of helium burning. The calculations show that the star goes through a series of low-amplitude relaxation oscillations (peak nuclear-energy generation $\approx 2.5 \times 10^{4} L_{\odot}$ ). The amplitude of these oscillations increase very slowly. Eventually the helium-burning shell, which is overtaking the hydrogen shell, stops burning because the temperature inside the helium shell falls below that necessary to maintain helium burning. At this point in the star's evolution the rate of hydrogen burning increases sharply until almost the entire luminosity of the star $\left(\approx 2200 L_{\odot}\right)$ is supplied by hydrogen burning. The helium layer beneath the hydrogen contracts and consequently the density inside the helium shell increases. As the hydrogen shell advances toward the surface the mass of the helium layer increases and eventually the temperature inside the helium shell begins to rise. Helium burning commences and after the helium-burning shell has reached about $100 L_{\odot}$ it becomes unstable again.

At the onset of the instability the density within the helium burning shell is $\approx 3 \times 10^{4} \mathrm{gm} / \mathrm{cm}^{3}$ as compared with $\approx 9 \times 10^{3} \mathrm{gm} / \mathrm{cm}^{3}$ encountered during the earlier low-amplitude relaxation oscillations. The highest rate of nuclear-energy generation attained during the final oscillation is $\approx 3 \times 10^{6} L_{\odot}$ or approximately $10^{2}$ times higher than is attained during the earlier relaxation oscillations. In addition, the helium convective zone associated with the final relaxation oscillation extended to a mass shell very close to the hydrogen shell so that mixing nearly took place. Hayashi et al. (1965) have discussed solutions where an extinguished helium shell is turned on again in a manner similar to what has been described above. In carrying out their solutions they assumed that the core is isothermal and that the shell must be turned on under degenerate conditions. In our case the helium shell goes out for the final time after one moderately-large amplitude oscillation. However, if the hydrogen-rich envelope of our star were more massive, the helium shell might have been turned on again under conditions more favorable for large amplitude oscillations.

The above discussion of unstable models may be summarized by saying that the calculations indicate that a thermally unstable star undergoes a series of relaxation oscillations that are of insufficient amplitude to cause any significant acceleration of mass. These initial relaxation oscillations are followed by a much smaller number (perhaps only one) of much more violent relaxation oscillations that may become important hydrodynamically.

O’Dell (1963) and Seaton (1966) have estimated the luminosities and effective temperatures for a number of planetary nuclei (see also Osterbrock, 1966). From the observed expansion velocities and estimated diameters for the nebulae it is possible to obtain a time-scale for their evolution if it is assumed that the nuclei represent a 
reasonably well-defined evolutionary track. The observations indicate that this assumption is justified. In a previous paper (Rose, 1967a) the final relaxation oscillation of a $0.75 M_{\odot}$ star has been compared in the H-R diagram with the results of Seaton for the estimated tracks of observed central stars. These calculations indicate that it is possible to understand the high luminosities and short lifetimes estimated for these central stars if a large amplitude thermal instability has taken place at the time of the formation of a planetary nebula.

The computed models with pure helium envelopes are bluer than most of the observed nuclei of planetary nebulae. However, models of the final relaxation oscillation of the $0.6 M_{\odot}$ sequence, which contains a $0.003 M_{\odot}$ hydrogen-rich envelope at this stage of its evolution, are much redder than these nuclei. This is true because of the low molecular weight of hydrogen as compared with helium. The effect of the hydrogen on the radii is made more pronounced because of the high luminosities of the models. On the basis of these calculations it can be concluded that the mass of hydrogen-rich matter in typical central stars is $<0.003 M_{\odot}$. For some nuclei it must be considerably less than this value. Therefore, the very blue colors of central stars suggest that whatever the cause of planetary nebulae the energy source is beneath the hydrogen shell.

The observations suggest that the maximum luminosity of the central star does not occur immediately following the formation of the nebula but perhaps several thousand years later. It has been shown by computed models (e.g. Rose, 1966) that a decrease in surface luminosity occurs during the interval of high nuclear-energy generation if the thermal instability is sufficiently violent. More recent calculations of $0.6 M_{\odot}$ models described above show that during the final relaxation oscillation the surface luminosity decreases from $\approx 2200 L_{\odot}$ to $\approx 200 L_{\odot}$ before increasing to higher luminosity $\left(\approx 4500 L_{\odot}\right)$ after the photons that were emitted during the thermal instability have had time to diffuse to the surface. The time between the model with the highest nuclear-energy generation $\left(\approx 3 \times 10^{6} L_{\odot}\right.$ for this case) and the model with the highest surface luminosity is $\approx 10^{3}$ years. The observational evidence for relatively low luminosity for central stars during the very early stages in the formation of planetary nebulae is not very strong. However, if future observations confirm the effect it would provide evidence for the turning on of a nuclear source at approximately the time a planetary nebula is formed.

Eddington (1926) first pointed out that nuclear-energy generation can lead to pulsational instability in a star. However, most stars are known to be pulsationally stable with respect to excitation by nuclear sources. This is true primarily because the modes of oscillation are usually non-homologous i.e. the relative amplitudes of all the fundamental modes of oscillation are much higher near the surface layers where radiative dissipation usually dominates than in the interior region of the star where nuclear sources are situated. As a consequence, the stabilizing influence of radiative dissipation is usually sufficient to overcome the destabilizing influence of nuclearenergy generation. However, it has been known for some time that very massive stars 
(Ledoux, 1941 ; Schwarzschild and Härm, 1959) and certain white dwarfs (Ledoux and Sauvenier-Goffin, 1950; Lee, 1950) ought to become pulsationally unstable. More recently it has been shown that thermal instability can lead to pulsational instability in a blue star (Rose, 1967a, $b$ ). It has been suggested that the occurrence of novae and certain X-ray sources may result because a thermal instability has caused a blue star to become pulsationally unstable. On the other hand, it has been argued that the progenitors of planetary nebulae are most probably red giants. For stars with extended envelopes the relative amplitude of the adiabatic modes of oscillation will be much smaller in the region of helium burning than in the extended convective envelope and therefore it is extremely difficult to excite adiabatic modes. However, for the very high rates of nuclear-energy generation, such as result from very large amplitude relaxation oscillations, the $e$-folding time for the development of the thermal instability will be less than the time for sound to traverse a red giant and so an injection of nuclear energy of $\approx 10^{49}$ ergs is expected in a time-scale that is less than the red-giant fundamental pulsation period $\left(\approx 10^{7} \mathrm{sec}\right)$. It follows that for this case, which appears to be the case of interest as far as the formation of planetary nebulae is concerned, the results of adiabatic pulsation theory are not adequate. It remains to be shown that the amplitude of a relaxation oscillation can become sufficient to lead to non-adiabatic pulsational instability and mass loss.

The calculations that have been carried out so far do not make it appear probable that the input of nuclear energy will become sufficiently rapid to lead directly to the formation of a shock wave. A shock wave will be formed in the burning region only if the pressure varies significantly in less than 1 second.

It should be pointed out that a thermal instability associated with a helium-burning shell may lead to the mixing of hydrogen from the envelope into the hotter interior regions (Schwarzschild and Härm, 1967). In the calculation of Schwarzschild and Härm only a very small amount of hydrogen was mixed into the hot interior. However, if it could be shown that as much as $\approx 10^{-3} M_{\odot}$ of hydrogen were mixed into the helium zone then as much as $10^{49}$ ergs might be released by means of the $\mathrm{CN}$ cycle in several convective mixing times scales $\left(\approx 3 \times 10^{5} \mathrm{sec}\right)$. It may turn out that this is more favorable mode of releasing nuclear energy rapidly than by means of helium burning directly.

We summarize the arguments concerning the origin of planetary nebulae that have been presented in this paper:

(1) The lifetimes and luminosities observed for the nuclei of planetary nebulae can be explained even for nuclei with low mass.

(2) The observed very blue colors of central stars indicate that typical planetary nuclei have $<\frac{1}{2} \%$ hydrogen by mass. Some nuclei must have negligible hydrogen. This suggests that the energy source for the formation of planetary nebulae is below the hydrogen-burning shell.

(3) The maximum luminosity for planetary nuclei appears to come sometime after 
the formation of the nebula. This can be understood as due to the turning on of a nuclear source.

(4) Instabilities associated with helium burning appear to arise as a direct consequence of stellar evolution.

\section{References}

Abell, G., Goldreich, P. (1966) Proc. astr. Soc. Pacific, 78, 232.

Christy, R. (1966) Astrophys. J., 144, 108.

Eddington, A. (1926) The Internal Constitution of the Stars, Cambridge University Press, Cambridge.

Faulkner, J. (1966) $\quad$ Astrophys. J., 144, 978.

Faulkner, J., Iben, I., Jr. (1966) Astrophys. J., 144, 995.

Hayashi, C., Hoshi, R., Sugimoto, D. (1962) Progress. theor. Phys., Suppl. 22.

Hayashi, C., Hoshi, R., Sugimoto, D. (1965) Progress. theor. Phys., 34, No. 6.

L'Ecuyer, J. (1966) Astrophys. J., 146, 845.

Ledoux, P. (1941) Astrophys. J., 94, 537.

Ledoux, P., Sauvenier-Goffin, E. (1950) Astrophys. J., 111, 611.

Lee, T.D. (1950) Astrophys. J., 111, 625.

O'Dell, C.R. (1963) Astrophys. J., 138, 67.

Osterbrock, D.E. (1966) in Stellar Evolution, Ed. by L.F. Stein and A. G.W. Cameron, Plenum Press, N.Y., p. 381.

Rose, W.K. (1966) Astrophys. J., 146, 838.

Rose, W.K. (1967a) Astrophys. J., 150, 193.

Rose, W.K. (1967b) Astrophys. J. (in press).

Schwarzschild, M., Härm, R. (1959) Astrophys. J., 129, 637.

Schwarzschild, M., Härm, R. (1965) Astrophys. J., 142, 855.

Schwarzschild, M., Härm, R. (1966) Private Communication.

Schwarzschild, M., Härm, R. (1967) Astrophys. J., 150, 961.

Seaton, M. (1966) Mon. Not. R. astr. Soc., 132, 113.

Shklovsky, I. (1956) Astr. Zu., 33, 315.

Weigert, A. (1965) Mitt. astr. Ges., p. 53.

\section{DISCUSSION}

Kippenhahn: In his models Rose has a carbon core and helium envelope. This would mean that his star must have already undergone mass loss. If, on the other hand, you want to get your pulsational instability in the red-giant region, you must put on top of your models a hydrogen envelope which is geometrically very thick. This would mean that the pulsational amplitudes will decrease rapidly inwards and will be fairly small at the region of the shells where the thermal pulses occur. Then the damping of the envelope might overcome the destabilizing effect of the shells. How will you overcome this difficulty?

Savedoff: Consider two cases: (a) mass loss by radiation pressure, (b) mass loss by limiting amplitude pulsations. When the radiation-pressure gradient is balanced gravitationally the gas expands into vacuum essentially at the speed of sound (Burger's expansion). Presumably release pressure will limit nuclear-energy rate. When pulsations build up to the escape velocity, the rapid energy loss will again maintain amplitude near the limiting value because of the tremendous energy drawn.

Rose: For the mechanism for the formation of a planetary nebula that I have discussed the energy input may take place in a time comparable with the time required for sound to traverse the red-giant envelope. Therefore, it may be possible to eject the envelope in a single shell.

In the evolution of the unstable $0.75 M_{\odot}$ star that I have discussed only the final relaxation oscillation was sufficiently violent to approximately explain the short time-scale and high luminosity that are observed for the nuclei of planetary nebulae. 